\title{
From Kegel exercises to pelvic floor rehabilitation: A physiotherapeutic perspective
}

\section{De los ejercicios de Kegel a la rehabilitación del piso pélvico: una visión a partir de la fisioterapia}

\author{
María Cristina Rodas, ${ }^{1}$ Herney Andrés García-Perdomo ${ }^{2}$
}

\begin{abstract}
Different pelvic floor therapy strategies have been adopted by physicians, urologists, gynecologists, and physiotherapists. However, the concept of pelvic floor physiotherapy is not yet clear for the majority of professionals, who continue to suggest activities that can actually be more harmful to the patient than beneficial. Consequently, the aim of this review article was to present the concept of pelvic floor physiotherapy, from its origins to its current principles, so that healthcare professionals can apply the therapy to their patients based on a clear understanding of the approach.

KEYWORDS: Pelvic floor therapy; Physiotherapy.

Resumen

La terapia del piso pélvico se ha indicado con diferentes estrategias, adoptadas por los médicos, urólogos, ginecólogos y fisioterapeutas. Sin embargo, el concepto de fisioterapia del piso pélvico aún no es claro para la mayoría de los profesionales, pues algunos protocolos sugieren actividades que pueden generar más daños que beneficios. En consecuencia, este artículo expone el concepto, desde sus orígenes hasta los principios actuales, que todos los profesionales deben conocer $y$, por lo tanto, aplicar en sus pacientes.

PALABRAS CLAVE: Piso pélvico; fisioterapia.
\end{abstract}

\author{
'PT, Pelvic Floor Physical Therapist, Pro- \\ fessor, Universidad del Valle, Colombia. \\ ${ }_{2}^{2}$ MD MSc EdD PhD FACS Associate Pro- \\ fessor, Universidad del Valle, Colombia. \\ Recibido: agosto 2018 \\ Aceptado: septiembre 2018 \\ Correspondencia \\ Herney Garcia Perdomo \\ Herney.garcia@correounivalle.edu.co \\ Twitter: @herneygarcia \\ Este artículo debe citarse como \\ Rodas MC, García-Perdomo HA. From \\ Kegel exercises to pelvic floor re- \\ habilitation: A physiotherapeutic \\ perspective. Rev Mex Urol. 2018 sept- \\ oct;78(5):402-411. \\ DOI: https://doi.org/10.24245/rev- \\ mexurol.v78i5.2472
}

\section{INTRODUCTION}

The pelvic floor is a set of structures, muscles, and connective tissues that provides the pelvic organs, such as the bladder, vagina, uterus, and rectum, with stability and support. It may more frequently be affected or weakened in women by the factors of pregnancy, childbirth, and menopause, or by poor micturition habits, constipation, high-impact sports, or professional activities, among others. As a result, the so-called pelvic floor dysfunctions can occur, which include (but are not limited to) urinary incontinence, fecal incontinence, sexual problems, pelvic pain, and genital prolapse. In the late 1940s, the gynecologist Arnold Kegel introduced pelvic floor exercises for women who presented with urinary incontinence. That approach pro- 
duced progressive contraction of the levator ani under strict supervision and introduced the concept of biofeedback, as well. ${ }^{1-3}$

Pelvic physiotherapy has currently undergone exponential development and plays an important role in the treatment of such disorders in men, women, and children. ${ }^{4}$ Pelvic floor muscle training, biofeedback, electrotherapy, vaginal cones, hypopressive abdominal techniques, and urotherapy are among the physiotherapeutic methods that specifically pertain to the pelvic floor. ${ }^{5}$ The comprehensive treatment of lumbopelvic problems and those of the perineal complex has demonstrated good results, improving the self-esteem and quality of life of patients. ${ }^{6}$ The aim of the present review was to describe the current developments that the Kegel exercises have brought to pelvic floor therapy.

\section{Kegel exercises}

In 1948, as a professor of obstetrics and gynecology in the United States, Dr. Arnold Kegel was the first to propose training of the pelvic floor muscles through a series of regularly repeated exercises. Their effectiveness was demonstrated in a paper titled Progressive resistance exercise in the functional restoration of the perineal muscles. ${ }^{7,8}$ In that study, 64 patients received treatment for managing pelvic dysfunctions, as well as stress urinary incontinence (SUI) and immediate postpartum and genital prolapse (cystocele and rectocele-older nomenclature).

In the context of the Kegel study, the levator ani is a striated muscle, consisting of $67 \%$ slow-twitch fibers and 33\% fast-twitch fibers, and its function is to squeeze around the openings of the anus, vagina, and urethra. Its contraction is not visible and is performed in a cephalad direction. ${ }^{9}$ The first biofeedback process was designed by Kegel, using a rubber electrode of approximately 3.5 inches. It recorded a pressure of 0-100 $\mathrm{mmHg}$.
The reading varied, according to the position in which it was performed. Most important was the correct performance of the levator ani, without including the abdominal or gluteal muscles. Patients were given directions on how to carefully perform the exercises, ${ }^{2}$ as well as how to overcome that contraction, and even how to increase its frequency for periods of 20 minutes, three times/day, reaching five contractions every 30 minutes. ${ }^{1,3}$ Dr. Kegel and his wife manufactured the necessary equipment in their kitchen and it was marketed in that fashion for approximately 30 years and up to three years after Dr. Kegel's death. The exercises are a simple method that can help improve the tone and function of that musculature. ${ }^{3,10}$

The Kegel article described how the patients were instructed to perform pubococcygeus muscle contractions. The first step consisted of recognizing the anatomic structures (the first Kegel exercise). The second step consisted of gentle digital exploration to verify the contraction and tone of the pubococcygeus muscle and other structures, and thus determine whether the patient knew those muscles had contracted. Finally, the third step was to assess whether that muscle contraction was learned. ${ }^{1}$

In the 1960s and early 1970s, the management indicated for urinary incontinence was surgical. ${ }^{11}$ The recommended first-line treatment, with no adverse effects, was the training of the pelvic floor muscles, but learning how to contract them correctly was not easy, and the verbal or written directions proved to be insufficient, as Kegel had found in $40 \%$ of the patients. ${ }^{12}$ To facilitate learning how to perform the exercises, the patient was instructed to imagine preventing the exit of fecal matter or urine flow. In 50\% of cases, that contraction was performed using accessory muscles of the abdomen or the gluteus, and in many cases worsened the symptoms. ${ }^{10}$ 
In his original publication in 1950, Kegel stated that the difference in contraction-relaxation of the pubococcygeus was $40 \mathrm{mmHg}$. Two years later, in 1952, he showed that the difference was 20 $\mathrm{mmHg}$, which was more realistic. ${ }^{2}$ Subsequent studies reported that the pressure of the vagina, in a healthy woman, was $25 \mathrm{mmHg}$ and could reach $45 \mathrm{mmHg}$ with a voluntary muscle contraction. Unfortunately, no further publications were published regarding the perineometer. ${ }^{2}$

Subsequently, a group of medical specialists found that the contraction of those muscles was related to orgasmic capacity, as discussed in studies by Kline-Graber and Graber (1978), and later by Masters and Johnson (1966). Finally, in 1972, Hartman and Fithian introduced the use of the perineometer as a tool that could assist in the treatment of sexual dysfunction, in accordance with the study by Kegel, Masters, and Johnson. ${ }^{2}$

Physical therapy as support for the pelvic floor

Within the management of pelvic floor dysfunction, the physiotherapist plays a very important role in prevention and treatment. His or her position is to inform patients about the dysfunction and changes in lifestyle and to employ physiotherapeutic resources. They include technical manuals and the training of the pelvic floor muscles with the help of biofeedback, electrical stimulation, and vaginal cones, among other techniques. The educational approach facilitates learning proper contraction and relaxation, with the goal of improving muscle strength and tone. Different programs are used, depending on the condition and clinical status of each patient. ${ }^{13,14}$

New procedures that act as a complement to Professor Kegel's exercises have been developed. Around 1970, studies by Magnus Fall, in Sweden, identified the use of electrical stimulation in the treatment of pelvic floor dysfunctions. ${ }^{14}$ In 1980,
Bourcier disclosed those perineal re-education techniques for treating urinary incontinence in written press and audiovisual media, and was recognized as a great promoter of perineal reeducation. ${ }^{14}$ Since then, different professionals have been interested in the pathophysiologic, epidemiologic, preventive, and therapeutic aspects of pelvic floor dysfunctions, leading to the development of conservative noninvasive therapy for the clinical conditions of incontinence, genital prolapse, neurologic lesions, sexual dysfunction, and anorectal canal dysfunction in men, women, and children. ${ }^{15}$

In 1992, the International Continence Society (ICS) validated employing those techniques for the treatment of perineal disorders and the functional recovery of the pelvic floor. ${ }^{14}$ The techniques are evidence-based, which aids the physiotherapist in developing a treatment plan based on clinical research. Several studies have shown the interventions to be effective in the management of SUI, but there is still insufficient evidence in relation to other dysfunctions. ${ }^{13}$

The pelvic physiotherapist evaluates the patient and establishes a physiotherapeutic diagnosis, which enables a protocol and a non-invasive treatment modality to be organized. The first step is to educate the patients, to establish a strategy, and to formulate a preventive program and a supplemental protocol that provide positive reinforcement for progressing in their recovery. Hence, the physiotherapist-patient interaction is very important in this management. The second step is to inform about, understand, and evaluate the function of the perineal muscles and the correct contraction and relaxation forms, as well as to provide accompaniment during all phases of treatment. The final step is maintenance of and adherence to the treatment, enabling its proper performance during daily activities, with good perineal awareness. ${ }^{16}$ 


\section{Pelvic floor muscle training}

With respect to the training of the perineal muscles, we must not forget that they are skeletal muscles, and therefore, their proper assessment and the establishment of certain therapeutic objectives facilitate muscle training, which is based on two concepts: improving urethral resistance and improving pelvic organ support. ${ }^{8,17}$

Such training is posited to lead to increased muscle strength, tone, and resistance, along with improved neuronal aspects, producing morphologic and other specific changes in the long term and resulting in automatic contraction of that musculature. ${ }^{8}$ All of those changes depend on the training program and the type of exercise. In addition, even genetic and hereditary factors can have an influence. The aim is to reach a point where the levator ani is characterized by having type I (slow-twitch) fibers and type II (fast-twitch) fibers (Ila/llb), ${ }^{14}$ with a powerful baseline tone, which is necessary for avoiding prolapses and both urinary and fecal incontinence. . $^{13,18}$

The previous muscle training helps to normalize muscle-aponeurosis tension, achieving a balance between the abdominal/pelvic cavity and respiratory dynamics, the performance of the exercises, and the different postures that are adopted, all of which are important to keep in mind with this type of therapy. ${ }^{19}$ It has also been shown to be beneficial in the management of sexual dysfunctions. ${ }^{14}$

When a maximum voluntary contraction is demanded, the greatest number of muscle fibers are recruited so that an exercise can be performed. That muscle fiber recruitment is termed maximum muscle force, and the following must be considered:

Muscle power or maximum force (power): explosive aspectStatic resistance (endurance): for a specific period, maintaining an optimal isometric contraction (submaximal force).

Dynamic resistance (repetitions): dynamic endurance, repeating an optimal contraction a specific number of times. ${ }^{18}$

In the physiotherapeutic evaluation, hypertonia of the vaginal walls can be found, requiring manual techniques, relaxation exercises, breathing exercises, flexibility, and awareness. ${ }^{19}$ Another important recommendation is motivating the patient to perform the exercises while carrying out daily activities. ${ }^{19}$

Initially, the sessions can be conducted individually, thereby facilitating a greater understanding of the exercises and therapist-patient interaction. ${ }^{14}$ Later, they can be performed in a group, for example, women who are postmenopausal, postpartum, or women that share other similar characteristics. The easiest position for performing the exercises is the supine/prone position because there is no effect of gravity. Subsequently, to the extent that strength is gained, the difficulty of the exercises can be increased by performing them in positions against gravity or by simulating the exertions of common daily acitivites. ${ }^{14}$

The treatment can be performed two or three times a week, for a minimum of three months, thus achieving muscle hypertrophy and good muscle strength. We must not forget that the ability to perform a contraction is greater, the more relaxed and stretched the muscle is, and in turn, emphasize to the patient the importance of relaxing the muscles. ${ }^{14}$

When patients are asked to carry out a voluntary contraction of the pelvic floor, $30 \%$ do not perform the contraction or they perform a Valsalva maneuver. ${ }^{8}$ An aspect that should be considered is the role of the diaphragm, the abdominal 
muscles, and the intra-abdominal pressure on the pelvic muscles. ${ }^{20}$ Those phases of training follow a sequence: knowledge of the perineal area and the function of the levator ani muscle, awareness and use of the agonist and antagonist muscles, an increase in muscle strength, and perineal automatism while carrying out daily activities. ${ }^{14}$

Kegel recommended 300-400 contractions daily. In practice, a lack of adherence to and the abandonment of that practice have been demonstrated. Several authors have concluded that quality is better than quantity, in reference to muscle contraction. ${ }^{18}$ Special attention should be given to muscle function and coordination and to awareness of the pelvic contraction and urethral closure during physical activity. Techniques such as the perineal block, perineal co-contraction, or the Knack maneuver become necessary as a preliminary action before signs of abdominal hyperpressure present. ${ }^{21}$

In general, pelvic floor muscle training is not performed in isolation and can be combined with other techniques, such as electrotherapy and biofeedback. ${ }^{19}$

Different protocols have been used in all the studies evaluating pelvic floor muscle training programs, making it impossible to conclude which is the most effective. ${ }^{17}$

\section{Physiotherapeutic techniques used in rehabilitation}

The commencement of any educational modality requires a complete evaluation that includes the anamnesis, through which the reason for the consultation is known, as well as the patient's urologic, gynecologic, sexual, and pelvic pain histories, lifestyle, and daily habits, among other factors. ${ }^{15}$

Whichever technique is used, it is essential to thoroughly explain to the patient the anatomy involved, the treatment objectives, and the treatment plan. Said approach facilitates the use of various therapeutic modalities, whether through the application of instrumental techniques or behavioral management. That information should be reinforced throughout the entire process of perineal education, furthering the integration of the pelvic muscles in the performance of daily activities. ${ }^{15}$

The patient's ability to contract the pelvic musculature is confirmed through muscle assessment and the physiotherapist may use different methods. A manual test (digital palpation) is a simple subjective method that is not a scientific evaluation. The Oxford scale and the PERFECT test are used for both the assessment and treatment of pelvic dysfunctions ${ }^{14}$ and they evaluate muscle strength, static resistance or endurance, and dynamic resistance or fatigability.

Body awareness (awareness) is the most important and delicate part of the physiotherapeutic evaluation, ${ }^{14}$ in which the physiotherapist has the task of influencing the awareness of the patient. It often takes several sessions to perform the correct contraction, which is difficult to achieve during the first consultations. Once that is achieved, the physiotherapist then moves forward to the stage of muscle training, which is one of the most important. ${ }^{14}$

\section{Biofeedback or feedback}

Because of the many benefits it confers, biofeedback is currently the method most widely used in the training of the pelvic musculature during perineal rehabilitation. ${ }^{17}$ Arnold Kegel (1959) was the first to present manometric biofeedback. Through the introduction of an electrode, the technique assesses the pressure exerted by the vagina. The electrode is connected to a manometer, which records the pressure change when the musculature of the perineum moves. 
The recording is measured in centimeters of water. ${ }^{18,22}$ However, it can reveal a contraction of the perineum resulting from abdominal hyperpressure, which is counterproductive for its use as a learning medium. ${ }^{18}$ Manometric biofeedback is low-cost and easy to use. By inflating a balloon, it can work at different pressure levels, based on perineum muscle strength. Both physical and physiologic alterations, such as temperature, vaginal mucosa, hormonal disturbances, the latex material of the electrode, or abdominal pressure variations, can alter the recording. ${ }^{14}$ The procedure is able to provide a graph of both the phasic and tonic muscle fibers, which may vary according to the inflation of the balloon. ${ }^{14}$

The technique of biofeedback with electromyography employs more sophisticated equipment, can be vaginal or anal, and uses either surface electrodes or intracavitary probes, which in the case of the pelvic floor muscles, produce a better recording of the action potential, as well as providing an electromyographic record of the muscles. ${ }^{14}$ The image captured on the computer screen by the physiotherapist corresponds to the myoelectric activation of the muscle, representing a physiologic or pathophysiologic phenomenon. The action potential is transmitted through the nerve fiber as a nerve impulse that is captured by the electrodes being used on the patient (the electrodes are either surface electrodes, intracavitary electrodes, or electrodes with needles). The recorded electrical disturbance will be the sum of all action potentials produced by the muscle fibers activated at that time. The recording of the action potential, in microvolts, shows which motor units are being recruited, as demonstrated by the curve in terms of frequency and amplitude. The modern equipment makes the mathematical correction, without losing recording sensitivity. Occasionally, there is interference that may show the wrong information, so the computer uses filters that assist in its management. ${ }^{14,19}$ Biofeedback is of great use. It is an active technique, which ensures the participation of patients in their rehabilitation, ${ }^{15}$ and is an excellent method for helping patients identify their pelvic floor, without the activity of other muscle groups, such as the abdominal and adductor muscles. It is necessary to use a computer, which facilitates the objective awareness of an unconscious physiologic function using a visual or auditory signal and an experienced professional, concurrently increasing the time and cost of the treatment. ${ }^{23}$ Biofeedback is also useful for motivating and assessing learning progress, giving positive or negative reinforcement or, if desired, activating the maximum and efficient contraction of the pelvic floor, as would be the case when there are symptoms of urinary urgency. ${ }^{14,19,21}$

There are certain characteristics that may be useful in the selection of the patients that are good candidates for receiving care through biofeedback, such as motivation, IQ, inability to voluntarily contract/relax their pelvic floor muscles, or a very low contraction capacity, and the use of imaging techniques and body awareness can also be of substantial help. The expectations that are met in the patient's recovery will be determined by all those factors. ${ }^{19}$

In general terms, the objectives should focus on training the patient to identify the pelvic floor muscles; on evaluating and normalizing muscle tone, which will facilitate correct and efficient muscle contraction; on improving and increasing perineal perception, preventing or controlling the contraction of accessory muscles; and finally, on allowing the physiotherapist to receive feedback in response to a proposed training plan. ${ }^{19}$

\section{Electrical stimulation}

Early uses of electrical stimulation in pelvic reeducation were performed by Magnus Fail in Sweden for the treatment of irritative bladder 
symptoms. ${ }^{14,19,24}$ In 1983, McGuire published studies in which transcutaneous electrical stimulation was applied to the posterior tibial nerve, and in 1987, Cooperberg and Stoller modified the technique by using percutaneous electrodes with acupuncture needles. ${ }^{19,25}$

Electrical stimulation can be applied as neurostimulation, stimulating the pudendal nerve (efferent fibers), thus producing a muscle contraction. It can also be applied indirectly to inhibit the detrusor muscle through the stimulation of the afferent fibers of the pudendal nerve. ${ }^{17}$

At present, electrical stimulation is used to treat pelvic dysfunctions, such as urinary and fecal incontinence. It facilitates contraction of the pelvic musculature in a passive manner, enabling the patient to become aware of such contractions (proprioception), helping to improve the tone and strength of the contraction, inhibiting the bladder, and decreasing pain. ${ }^{18}$

The mechanism of action requires an intact spinal arc, so that the reflex activation can occur, which will produce a contraction in either the peri-urethral sphincter, anal sphincter, or all of the pelvic floor muscles. Transcutaneous electrical stimulation can be performed with surface electrodes in the perineal region, ${ }^{17}$ intravaginally, or intrarectally. ${ }^{14}$ Percutaneous electrical stimulation can also be performed through tibial nerve stimulation with acupuncture needles. ${ }^{17}$

Long-term histologic changes are produced in the muscle fiber, as a consequence of hypertrophy and tonification that have been produced in those muscles. ${ }^{18}$

Currently, there are many difficulties involved in the use of electrical stimulation to treat urinary incontinence. Different types of currents, such as alternating, bipolar, or interferential currents, can be used. ${ }^{14}$ The parameters to bear in mind are frequency $(\mathrm{Hz})$, pulse length, and their combination, which should be considered in relation to the desired goal of each patient. The cycles of contraction and relaxation are also programmed to prevent muscle fatigue. The intensity should be the maximum level tolerated by the patient, without producing pain. ${ }^{14}$ Many studies on electrical stimulation use different parameters and different criteria for the treatment of diverse pathologies because they influence the therapeutic effect and can be combined with other techniques, such as kinesiotherapy, showing better results than electrical stimulation, alone. ${ }^{14}$

\section{Vaginal cones}

In pelvic floor muscle strength training, vaginal cones of different weights are used as a form of biofeedback. ${ }^{26}$ They act by contracting the pelvic floor to prevent the cone from slipping out. They can be used in a standing position, while coughing or walking, for no longer than 15-20 minutes, as longer use can produce pain, decreasing blood flow and oxygen consumption. ${ }^{13}$ When to change to a heavier cone is a form of self-learning and motivation for the woman. Cone weight varies from $25 \mathrm{~g}$ to $75 \mathrm{~g}^{14}$ and they can be used without supervision or vaginal examination. The use of cones can help, but the inclination of the vagina in each woman will affect the equilibrium of the muscle contraction. ${ }^{26}$

Vaginal cones assist in the activation of type I and type II fibers, improving proprioception and muscle strength. ${ }^{11}$ They are also useful in training the patient to eliminate the Valsalva maneuver, because if it occurs, the cone falls out. Consequently, cone use helps the patient identify the perineal muscles and the muscles that act synergistically (such as the abdominal, adductor, and gluteal muscles) more precisely, improving proprioception. ${ }^{14}$ 
Treatment with cones aids in pelvic floor recovery, allowing the performance of isometric and isotonic contractions. At present there is no agreement regarding the form and duration of treatment. Some authors recommend that the cone be utilized during the patient's performance of her daily activities, as an active phase of treatment. ${ }^{14}$

Muscle training using vaginal cones offers good results in the management of SUI, according to comments by Kari Bo et al, who cite three randomized trials. The outcome was measured subjectively, with an evidence level of $1 .{ }^{18}$ In a Cochrane review, a study was conducted using vaginal cones in women with stress incontinence and those authors concluded that the training was more effective than not performing the treatment. ${ }^{26}$

\section{Behavioral therapy}

As conservative management, behavioral therapy includes a set of techniques that help to minimize urinary symptomatology, especially in patients with lower urinary tract symptoms. The therapy is recommended as a first-line treatment and includes changes in lifestyle or behavior, helping to reduce pelvic floor symptoms. ${ }^{17}$ Behavioral therapy includes three important aspects for managing urinary incontinece: daily micturition, education, and bladder training. ${ }^{14} \mathrm{~A}$ micturition diary should contain as much information as possible related to the intake and type of liquids, time of micturition, and episodes of incontinence, among other events. Patients must be educated and offered a brief and simple explanation of anatomy and function, control of fluid intake, genital hygiene, bowel habits, postures to facilitate bowel movements. Finally, the goal of micturition training is to improve bladder capacity, programming a micturition schedule-hence, the importance of knowing the patients' routine, life habits, medication use, etc. ${ }^{14}$ For the phys- iotherapist, knowing other modifiable factors, such as obesity, smoking, physical activity, and constipation, will greatly assist in the management of pelvic floor dysfunctions. ${ }^{17}$

\section{Manual therapy}

The use of manual therapy through muscle or joint manipulations can be useful in pelvic dysfunctions. Such manipulations are of great help in the management of scars, contractures, trigger points, or any other structure of the body that may affect the functioning of the pelvic floor, such as the diaphragm, lumbopelvic fasciae, and the vertebral column. Perineal massage (internal or external) can be performed before childbirth, an event in which soft structures, such as ligaments, fascia, and muscles, will be subjected to overstretching. Manual therapy includes the technique of digitopuncture, as well as the invasive technique of dry needling and myofascial release techniques for the handling of trigger points. Scars can be treated through scar massage techniques or combined with the application of ultrasound or some types of laser. ${ }^{18}$

\section{Abdominal gymnastics}

In the treatment of pelvic dysfunctions, the abdomen should be evaluated and trained. Abdominal muscles act synergistically with the perineal musculature. Consequently, it is important for the physiotherapist to assess whether synergistic activity of the pelvic muscles with the transverse abdominal muscle is occurring because that would facilitate bladder neck elevation and closure, increasing intraurethral pressure, thus controlling urinary leakage. ${ }^{18}$ Studies conducted by Sapsford and other authors have demonstrated that there is co-activation of those muscles during simple activities such as raising the arms. ${ }^{27}$

To train the abdominal muscles, hypopressive abdominal gymnastics (HAG) is used, as described 
by the physiotherapist Marcel Caufriez (1980), with the aim of improving the tone of the abdominal and perineal muscles, thus normalizing the tension of the musculo-aponeurotic structures and avoiding prolapse and urinary incontinence. That technique includes a series of global postures and hypopressive maneuvers, such as diaphragmatic breathing techniques, reflex neurofacilitation techniques, and hypopressive gymnastics. ${ }^{19}$

Another technique used to promote the synergy between the pelvic floor and the deep abdominal musculature, accompanied by self-stretching postures and postural control, has been described by Bernardett De Gasquet. No studies have yet demonstrated its effectiveness. Likewise, the physiotherapist Luc-Guillarme developed a technique for effective and synergistic abdominal contraction with active expiration, thereby protecting the pelvic floor from excessive intraabdominal pressure. The Pilates method is also based on a program of exercises that focus on strength, core stability, muscle control, flexibility, posture, and breathing. ${ }^{18}$

\section{CONCLUSIONS}

The initial stage of the comprehensive evaluation will allow the pelvic floor physiotherapist to decide which instrument or therapeutic aid will be the most effective for each patient.

The muscle training protocol must be specific for each patient, taking strength, resistance, and fatigability of the muscle fiber into account.

Education greatly facilitates therapeutic success because it makes positive feed-back of the performance by the patient possible.

\section{REFERENCES}

1. Gonzalez L, et al. Programa protocolizado de fortalecimiento del piso pelviano para la mujer adulta. Arch Gin Obs 2004;42(1):10.
2. Levitt $\mathrm{EE}$, et al. Intravaginal pressure assessed by the Kegel perineometer. Arch Sex Behav 1979;8(5):425-30.

3. Fitz FF, et al. Biofeedback for the treatment of female pelvic floor muscle dysfunction: a systematic review and meta-analysis. Int Urogynecol J 2012;23(11):1495-516. DOI: 10.1007/s00192-012-1707-1

4. Ladi Seyedian SS, et al. Combined functional pelvic floor muscle exercises with Swiss ball and urotherapy for management of dysfunctional voiding in children: a randomized clinical trial. Eur J Pediatr 2014;173(10):1347-53. DOI: 10.1007/s00431-014-2336-0

5. Mulders MM, et al. Urotherapy in children: Quantitative measurements of daytime urinary incontinence before and after treatment according to the new definitions of the International Children's Continence Society. J Pediatr Urol 2011;7(2):213-8. DOI: 10.1016/j.jpurol.2010.03.010

6. Dumoulin C, et al. Pelvic floor muscle training versus no treatment, or inactive control treatments, for urinary incontinence in women. Cochrane Database Syst Rev 2014;(5):CD005654. DOI: 10.1002/14651858.CD005654.pub3

7. Kegel A. Progressive resistance exercise in the functional restoration of the perineal muscles. Am J Obstet Gynecol 1948;56(2):238-48.

8. Krishna Dass A, et al. Diagnosis and conservative management of female stress urinary incontinence. Gynecol Minim Invasive Ther 2013;2(2):48-51. DOI: https://doi. org/10.1016/j.gmit.2013.02.005

9. Konstantinos $\mathrm{H}$, et al. Dilemmas in the management of female stress incontinence: the role of pelvic floor muscle training. Int Urol Nephrol 2007;38(3-4):513-25. DOI: 10.1007/s11255-006-0085-3

10. Vickers D, Davila GW. Kegel Exercises and Biofeedback. In: Davila G., Ghoniem G., Wexner S., editors. Pelvic Floor Dysfunction. London: Springer, 2008;303-10.

11. Silva AMN, Oliva LMDP. Exercícios de Kegel associados ao uso de cones vaginais no tratamento da incontinência urinária: Estudo de caso. Sci Med (Porto Alegre). 2011;21(4):1736. Dirección URL: <http://revistaseletronicas.pucrs.br/ojs/index.php/scientiamedica/article/viewFile/8982/7238>.

12. Bump RC, et al. Assessment of Kegel pelvic muscle exercise performance after brief verbal instruction. Am J Obstet Gynecol 1991;165(2):322-9.

13. Bo K, et al. Evidence Based-Physical Therapy for the Pelvic Floor. Churchill Livingstone: Elsevier, 2007;438.

14. Moreno A. Fisioterapia em Uroginecologia -. $2^{\mathrm{a}}$ edição. Moreno A, editor. Brasil: Manole, 2009;226.

15. Ibrahim I, et al. Efficacy of biofeedback-assisted pelvic floor muscle training in females with pelvic floor dysfunction. Alexandria J Med 2015;51(2):137-42. DOI: https://doi. org/10.1016/j.ajme.2014.06.001

16. Berghmans $B$. The role of the pelvic physical therapist. Actas Urol Esp 2006;30:110-22. 
17. $B \varnothing K$, et al. Evidence-based physical therapy for the pelvic floor: bridging science and clinical practice. Evidence-based Physical Therapy for the Pelvic Floor: Bridging Science and Clinical Practice. 2007. 1-8 p.

18. Ramirez Garcia I, et al. Rehabilitación del suelo pélvico femenino. García IR, editor. Bogota: Panamericana, $2013 ; 200$.

19. Palma P. Urofisioterapia Aplicações Clínicas das Técnicas Fisioterapêuticas nas Disfunções Miccionais e do Assoalho Pélvico. 2a ed. Palma P, editor. Campinas: Personal Link Comunicações Ltda; 2014. 576 p.

20. Park H, Han D. The effect of the correlation between the contraction of the pelvic floor muscles and diaphragmatic motion during breathing. J Phys Ther Sci 2015;27:2113-2115.

21. Burgio KL. Update on Behavioral and Physical Therapies for Incontinence and Overactive Bladder: The Role of Pelvic Floor Muscle Training. Curr Urol Rep 2013;14(5):457-64.
22. Rioja Toro J, González Rebollo a., Estévez Poy P. Pruebas de evaluación en la incontinencia urinaria femenina. Rehabil 2005;39(6):358-71.

23. Burgio KL. Behavioral Treatment of Urinary Incontinence, Voiding Dysfunction, and Overactive Bladder. Obstet Gynecol Clin North Am 2009;36(3):475-91.

24. Fall $M$, et al. Electrical stimulation in interstitial cystitis. J Urol 1980:123(2):192-5.

25. Cooperberg MR, Stoller ML. Percutaneous neuromodulation. Urol Clin North Am 2005;32:71-8.

26. Herbison P, Plevnik S, Mantle J. Conos vaginales pesados para la incontinencia urinaria. La Bibl Cochrane Plus. 2008;4.

27. Sapsford RR, et al. Co-activation of the abdominal and pelvic floor muscles during voluntary exercises. Neurourol Urodyn 2001;20:31-42.

\section{AVISO IMPORTANTE}

La Revista Mexicana de Urología se convierte en una publicación solo digital, con todas las ventajas que los medios y dispositivos electrónicos ofrecen. Usted podrá revisar la información mediante el sitio web (www.revistamexicanadeurologia.org.mx) o descargando la app para Android o iPhone.

Para consultar el texto completo de los artículos deberá registrarse por una sola vez con su correo electrónico, crear una contraseña, indicar su nombre, apellidos y especialidad.

Esta información es indispensable para saber qué consulta y cuáles son sus intereses, y poder en el futuro inmediato satisfacer sus necesidades de información. 